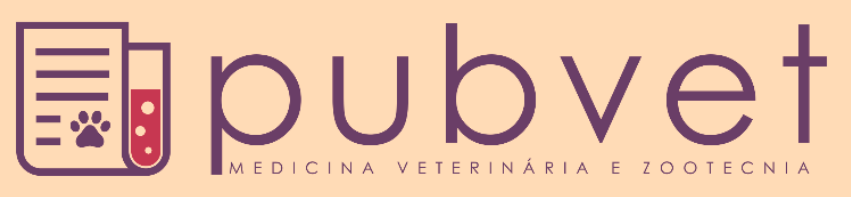

https://doi.org/10.31533/pubvet.v13n8a386.1-8

\title{
Comparativo e perfil dos infectados em esquistossomose no estado de Alagoas entre 2016 e 2017
}

\author{
Carlos Miguel Azarias dos $\operatorname{Santos}^{1 \bullet}$, Lyara Suzhanny de Oliveira Santos ${ }^{2} \bullet$, Jerffeson Araújo \\ dos Santos $^{20}$, Erilza dos Santos Silva ${ }^{2}{ }^{\circ}$, Maria Hilma dos Santos ${ }^{2}{ }^{\circ}$, Dayane Kelly Da Silva ${ }^{2} \bullet$ \\ , Josicleide Fernandes de Souza Santos ${ }^{3}{ }^{\circ}$, Douglas de Oliveira Subrinho ${ }^{\circ}{ }^{\circ}$, Cristiane Kelly \\ Aquino dos $\operatorname{Santos}^{50}$, Claudimary Bispo dos $\operatorname{Santos}^{60}$ \\ ${ }^{1}$ Graduando em ciências biológicas e pesquisador do grupo de extensão, Universidade Estadual de Alagoas - UNEAL $e$ \\ medicina veterinária, Faculdade Regional da Bahia - UNIRB, Arapiraca-AL Brasil.; \\ ${ }^{2}$ Graduanda do curso de ciências biológicas, Universidade Estadual de Alagoas - UNEAL, Arapiraca-AL Brasil., \\ ${ }^{3}$ Graduada em enfermagem, Centro Universitário Cesmac, Arapiraca-AL Brasil.; \\ ${ }^{4}$ Graduando em enfermagem, Centro Universitário Cesmac, Maceió-AL Brasil.; \\ ${ }^{5}$ Professora, pesquisadora, da Faculdade De Ensino Regional Alternativa - Fera curso de educação física e Faculdade \\ Regional da Bahia - UNIRB cursos da saúde, Arapiraca-AL Brasil. \\ ${ }^{6}$ Professora e pesquisadora, do Curso de ciências biológicas, Universidade Estadual de Alagoas - UNEAL, Arapiraca-AL Brasil. \\ *Autor para correspondência: carllos miguel1@ @otmail.com
}

\begin{abstract}
Resumo. A esquistossomose é uma doença parasitária causada por protozoário que tem como habitat rios, riachos e açudes que são comuns em Alagoas. Esse artigo visa analisar casos confirmados da esquistossomose em Alagoas e o perfil epidemiológico dos infectados durante os anos de 2016 / 2017. Será utilizada como metodologia a análise de dados estatísticos disponibilizados pelo Sistema de Informações de Agravos de Notificação (SINAN) e o embasamento teórico de publicações. Foram confirmados 125 casos positivos em 2016 e 70 casos em 2017, o perfil dos infectados no ano de 2016 foram 59\% feminino (F) e 41\% Masculino (M) em 2017 56\% M e 44\% F. Em números os casos em 2016 foram $74 \mathrm{~F}$ e $51 \mathrm{M}$ em $201739 \mathrm{M}$ e $31 \mathrm{~F}$. A maior faixa etária acometida em 2016 foi 20-59 anos e 2017 40-59 anos. Os municípios com maiores índices em 2016 é Joaquim Gomes, Novo Lino e Campo Grande,30, 15 e 12 e em 2017 Maceió, Campo Grande e Traipu, 22, 14 e 5 respectivamente. Totalizando 50 casos na zona urbana e 63 na rural e 12 em locais ignorados, 2017 apresentou-se 37 casos na urbana, 23 na rural e 10 em locais ignorados. Características socioeconômicas deixam Alagoas em altas condições de risco, a água destinada para consumo ou uso geral não é tratada, residências não possuem saneamento básico. É importante utilizar medidas preventivas no controle dos fatores de risco ambientais e educacionais para possam reduzir a quantidade de casos.
\end{abstract}

Palavras-chave: platelmintos, saúde pública, schistosoma

\section{Comparison and profile of those infected in schistosomiasis in the State of Alagoas in 2016 to 2017}

\begin{abstract}
Schistosomiasis is a parasitic disease caused by protozoa that has as habitat rivers, streams and dams that are common in Alagoas. This article aims to analyze confirmed cases of schistosomiasis in Alagoas and the epidemiological profile of those infected during the years 2016/2017. The methodology used will be the analysis of statistical data provided by the SINAN and the theoretical basis of publications. A total of 125 positive cases were confirmed in 2016 and 70 cases in 2017, the profile of those infected in the year 2016 was 59\% female (F) and 41\% Male (M) in $201756 \% \mathrm{M}$ and $44 \%$
\end{abstract}


F. In 2016 were $74 \mathrm{~F}$ and $51 \mathrm{M}$ in $201739 \mathrm{M}$ and $31 \mathrm{~F}$. The largest age group affected in 2016 was 20-59 years and $201740-59$ years. The municipalities with the highest indexes in 2016 are Joaquim Gomes, Novo Lino and Campo Grande, 30, 15 and 12 and in 2017 Maceió, Campo Grande and Traipu, 22, 14 and 5 respectively. Total of 50 cases in the urban area and 63 in the rural and 12 in the ignored places, 2017 presented 37 cases in the urban, 23 in the rural and 10 in ignored sites. Socioeconomic characteristics leave Alagoas in high risk, water intended for consumption or general use is not treated, households do not have basic sanitation. It is important to use preventive measures in controlling environmental and educational risk factors to reduce the number of cases.

Keywords: flatworms, public health, schistosoma

\section{Comparativo y perfil de los infectados en esquistosomiasis en el estado de Alagoas en 2016 a 2017}

Resumen. La esquistosomiasis es una enfermedad parasitaria causada por un protozoario que tiene como hábitat ríos, arroyos y azudes que son comunes en Alagoas. En este artículo se pretende analizar casos confirmados de la esquistosomiasis en el estado de Alagoas y el perfil epidemiológico de los infectados durante los años 2016 / 2017. Se utilizará como metodología el análisis de datos estadísticos disponibles por el Sistema de Información de Agravios de Notificación (SINAN) y el fundamento teórico de publicaciones. Se han confirmado 125 casos positivos en 2016 y 70 casos en 2017, el perfil de los infectados en el año 2016 fue 59\% femenino (F) y 41\% Masculino (M) en 2017 56\% M y 44\% F. En números los casos en 2016 fueron $74 \mathrm{~F}$ y $51 \mathrm{M}$ en $201739 \mathrm{M}$ y $31 \mathrm{~F}$. La mayor franja etaria acometida en 2016 fue 20-59 años y 2017 40-59 años. Los municipios con mayores índices en 2016 son Joaquim Gomes, Novo Lino y Campo Grande, 30, 15 y 12 y en 2017 Maceió, Campo Grande y Traipú, 22, 14 y 5 respectivamente. En total, 50 casos en la zona urbana y 63 en la rural y 12 en lugares ignorados, 2017 se presentaron 37 casos en la urbana, 23 en la rural y 10 en locales ignorados. Las características socioeconómicas dejan a Alagoas en altas condiciones de riesgo, el agua destinada para consumo o uso general no es tratada, residencias no poseen saneamiento básico. Es importante utilizar medidas preventivas en el control de los factores de riesgo ambiental y educativo para reducir la cantidad de casos.

Palabras clave: platelmintos, salud pública, schistosoma

\section{Introdução}

A esquistossomose é uma doença que envolve aspectos sociais, econômicos, culturais e políticos; não devendo ser vista como um problema individual ou de um grupo específico, mas dentro de um conjunto de fatores que são parte da vida da população de determinada região. De forma que para amenizar a situação devem-se melhorar diversas questões relacionadas, pois a endemia da parasitose é considerada por alguns como indicativo do nível socioeconômico da região (Jordão et al., 2014).

O ciclo biológico do Schistosoma mansoni é complicado, pois é composto por duas fases parasitárias: uma no hospedeiro definitivo (homem) e mais uma no hospedeiro intermediário (caramujo) (Uchoa et al., 2000). Existem, ainda, duas passagens de larvas de vida livre no meio aquático, que se mudam com as fases parasitárias. As fases evolutivas compõem no verme adulto (macho e fêmea), ovo, miracídio, esporocisto, cercária e esquistossômulo. O ciclo evolutivo do parasita encerra, em situações oportunas, em torno de 80 dias. No hospedeiro definitivo, o ciclo é sexuado e o tempo passado entre a penetração das cercárias e o encontro de ovos na excreção é em torno de 40 dias. No hospedeiro intermediário, o ciclo é assexuado e tem o mesmo período de aproximadamente, 40 dias (Katz \& Almeida, 2003).

Os vermes adultos estão inseridos nos vasos sanguíneos que conectam o intestino ao fígado do homem. Os machos são de coloração esbranquiçada e tem por medida entre 6 a $13 \mathrm{~mm}$ de comprimento por 1,1 mm de largura. A fêmea é cilíndrica e mais fina e longa que o macho. Tem por medida entre 10 a $20 \mathrm{~mm}$ de compridão por $0,16 \mathrm{~mm}$ de largura. Conforme não exibem órgão de copulação, a cópula acontece por aposição dos orifícios genitais dos femininos e masculinos, no qual as fêmeas estão 
instaladas no canal ginecóforo (fenda longitudinal, nos machos, para abrigar as fêmeas e fecundá-las) (Katz \& Almeida, 2003).

A transmissão da esquistossomose acontece quando entra em contato com águas contaminadas por cercárias, formato larval do parasito. Corriqueiramente nas partes endêmicas, os indivíduos usam rios habitados por caramujos infectados pelo Schistosoma mansoni, para banhos, pescas, lavagem de roupa e louças, estando suscetíveis à doença (Rocha et al., 2016).

A esquistossomose às vezes é confundida com outras enfermidades por causa das muitas manifestações clínicas que acontecem, a forma aguda frequentemente analisada em populações que não pertencem as áreas endêmicas. O exame parasitológico das excretas para diagnóstico dessa parasitose é executado essencialmente por meio dos métodos de sedimentação espontânea, entretanto Kato-Katz é o método padrão ouro preconizado pelo MS em regiões endêmicas (Rocha et al., 2016).

No Brasil, a esquistossomose se distribui em maior quantidade fortemente a cerca de alguma faixa de propriedades constantes e juntos, ao longo de aproximadamente inteira costa litorânea, acompanhando o caminho de consideráveis bacias hidrográficas (Saucha et al., 2015). O estado de Alagoas possui características específicas propícias para a disseminação da esquistossomose. Fatores sociais, culturais e naturais contribuem para que a doença permaneça entre as que mais acometem populações rurais e de periferias urbanas, trazendo consequências sanitárias e econômicas importantes para o comprometimento do desenvolvimento, sobretudo, humano do estado (SESAU, 2012). Atualmente, a disseminação da doença em Alagoas deve-se a existência de fatores endêmicos básicos para a viabilidade de todo o ciclo encontrado no estado. São eles: presença do hospedeiro susceptível (Homo sapiens sapiens); presença do hospedeiro intermediário (planorbídeos do gênero Biomphalaria); grande distribuição geográfica e alta resistência do hospedeiro intermediários à seca; presença de meios aquáticos adequados para a disseminação do hospedeiro intermediário no qual a população tenha contato direto, seja para atividades domésticas, agrícolas ou de lazer; depósito de esgoto nessas águas ou próximo a elas (Vitorino et al., 2012).

A doença está relacionada com a pobreza e com o baixo desenvolvimento econômico, levando a população a utilizar água proveniente de rios e açudes para suas atividades domésticas, atividades agrícolas e até para recreação, contribuindo de forma direta para a contaminação. Além disso, a ausência de tratamento de esgoto e de água, saneamento básico, acesso aos serviços de saúde e as baixas condições socioeconômicas são fatores determinantes para a endemia esquistossômica das regiões estudadas (Jordão et al., 2014).

A Esquistossomose mansoni é uma doença parasitária, causada pelo trematódeo Schistosoma mansoni, cujas formas adultas habitam os vasos mesentéricos do hospedeiro definitivo (homem) e as formas intermediárias se desenvolvem em caramujos gastrópodes aquáticos do gênero Biomphalaria. Com a grande proporção de casos positivos no estado de Alagoas no ano de 2017, visando entender quais fatores agravantes para aquisição dessa parasitose e analisar os fatores de riscos, observar a predominância de casos positivos de acordo com o sexo e faixa etária, identificar a zona de residência com mais números de casos e analisar os fatores de risco.

\section{Material e métodos}

A presente pesquisa foi desenvolvida através de um levantamento bibliográfico de dados disponível na plataforma virtual Sistema de Informações de Agravos de Notificação SINAN. (2017) referente a período 2016/2017, foram considerados os seguintes fatores: sexo; faixa etária e zona residencial, realizada no período de março a junho de 2018 no estado de Alagoas (Figura 1). A pesquisa epidemiológica documental pesquisada em plataforma digital por meio do Google Acadêmico no site SINAN. Podemos classificar o presente estudo como epidemiológico observacional do tipo ecológico. Tendo sido preconizada uma abordagem quantitativa e comparativa de caráter analítico de séries temporais (anos de registro), onde a associação entre o nível do fator de exposição e as taxas foi avaliada ao longo do tempo (Pereira, 1995). 


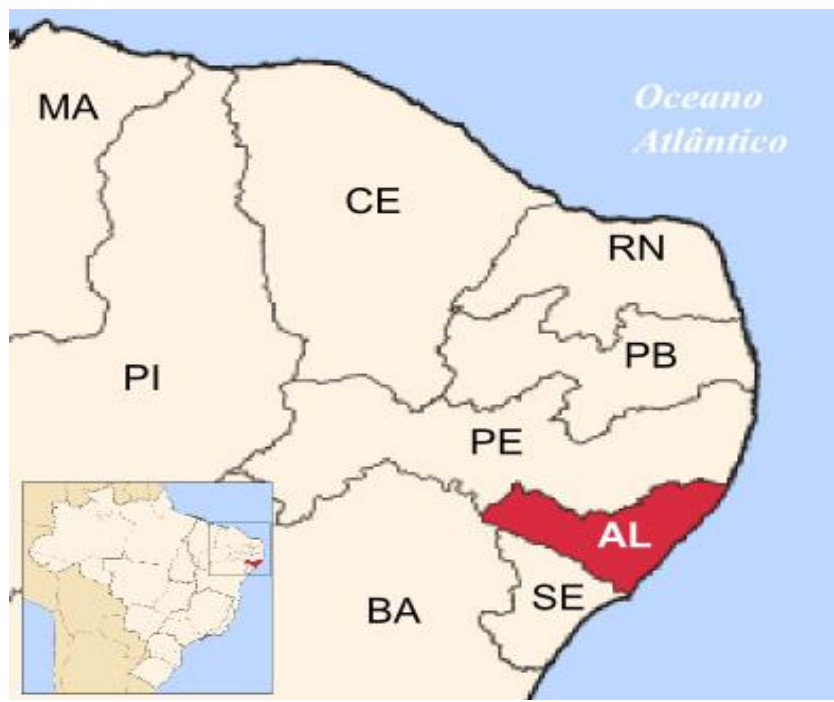

Figura 1. Estado de Alagoas. Fonte: Alves, Rodolfo

\section{Resultados e discussão}

No Brasil, a esquistossomose é uma doença endêmica de grande importância na saúde pública, com cerca de 43 milhões de pessoas vivendo em áreas de risco de infecção e sete milhões infectados (Rocha et al., 2016).

As tabelas 1 e 2 mostram a frequência da esquistossomose mansônica no Estado de Alagoas, de acordo com o percentual de positividade obtido por meio dos dados do Programa de Controle da Esquistossomose (PCE), no período de 2016. Observou-se que $59 \%$ dos casos positivos foram em indivíduos do sexo feminino enquanto, $41 \%$ apresentaram-se em indivíduos do sexo masculino, já em $201756 \%$ dos casos apresentaram-se em pessoas do sexo masculino e $44 \%$ no sexo feminino. A faixa etária com maior índice de casos positivos em 2016 foi entre 20-59 e em 2017 foi de 40-59 anos. Observou-se também que em 2016 a doença foi predominante em mulheres e 2017 em homens.

Levando em consideração os municípios alagoanos com maiores números de casos positivos no ano de 2016 foi Joaquim Gomes 30, Novo Lino, 15 e Campo Grande com 12. Durante 2017 Maceió liderou com 22, Campo Grande, 14 e Traipu com cinco casos.

Foi observado que em 2016 a zona mais acometida com pessoas infectadas foi a Zona rural com 63 casos de esquistossomose, na zona urbana registrou-se 50 casos e em locais ignorados 12 casos positivo, sendo os açudes e barragens o habitat do parasita. Já no ano de 2017 a prevalência de maiores números de casos foi na zona urbana com 37 casos, a zona rural com 23 e 10 em áreas não identificadas.

A exposição ao risco (contatos com a água) é pertinente principalmente entre pescadores e pessoas que moram em locais que não tem água encanadas a obtenção de água é feita diretamente em rios, lagoas ou represas que por sua vez são contaminadas e apresentam ambiente propício para o desenvolvimento das cercarias. A água não recebe nenhuma forma de tratamento antes de ser utilizada ou consumida. A pesca, principal atividade econômica praticada condiciona o uso contínuo das águas contaminadas e os moradores têm contatos diários prolongados. Diante desta exposição ao risco, é de se esperar uma alta prevalência da esquistossomose nestes locais.

Considerando as variáveis analisadas conjuntamente houve a prevalência de casos entre os indivíduos dos dois sexos, em idades economicamente ativa, ou seja, entre indivíduos que praticam a pesca ou alguma função equivalente em locais contaminados.

Neste artigo, a análise estatística dos fatores de risco selecionou variável e determinou a porcentagem de indivíduos que adquiriram a esquistossomose, mas a epidemiologia quantitativa possui limitações quanto à explicação causal dos fenômenos, associando, simplesmente, características individuais e doença, não levando em conta especificidades locais nem a natureza histórica e sociocultural dos eventos somáticos. Consequentemente, não há conhecimento suficiente para indicar medidas de saúde pública que possa modificar a qualidade de vida de grupos sociais menos favorecidos. 
Tabela 1. Notificação de casos em 2016 por município

\begin{tabular}{|c|c|c|c|c|}
\hline Município de notificação & Ignorado/Branco & Urbana & Rural & Total \\
\hline Anadia & - & 1 & 1 & 2 \\
\hline Barra de Santo Antônio & - & 1 & - & 1 \\
\hline Cacimbinhas & - & 1 & - & 1 \\
\hline Campo Grande & 1 & 6 & 5 & 12 \\
\hline Chã Preta & - & 1 & - & 1 \\
\hline Coité do Nóia & - & 1 & - & 1 \\
\hline Coruripe & - & 1 & - & 1 \\
\hline Dois Riachos & - & 1 & - & 1 \\
\hline Flexeiras & - & 1 & - & 1 \\
\hline Girau do Ponciano & - & - & 1 & 1 \\
\hline Ibateguara & - & 2 & - & 2 \\
\hline Jacuípe & - & 1 & - & 1 \\
\hline Joaquim Gomes & 2 & 1 & 27 & 30 \\
\hline Junqueiro & - & - & 2 & 2 \\
\hline Maceió & - & 8 & - & 8 \\
\hline Major Isidoro & - & - & 2 & 2 \\
\hline Maragogi & - & - & 2 & 2 \\
\hline Marechal Deodoro & - & 1 & 1 & 2 \\
\hline Matriz de Camaragibe & - & 1 & - & 1 \\
\hline Messias & - & 1 & - & 1 \\
\hline Novo Lino & - & 7 & 8 & 15 \\
\hline Olho d'Água Grande & - & - & 3 & 3 \\
\hline Palmeira dos Índios & - & 3 & 1 & 4 \\
\hline Pão de Açúcar & - & 1 & - & 1 \\
\hline Paripueira & - & 1 & - & 1 \\
\hline Pilar & - & 1 & - & 1 \\
\hline Porto de Pedras & - & - & 1 & 1 \\
\hline Porto Real do Colégio & - & 1 & 1 & 2 \\
\hline Quebrangulo & - & 1 & - & 1 \\
\hline Rio Largo & - & 1 & - & 1 \\
\hline Santana do Ipanema & - & 1 & - & 1 \\
\hline Santana do Mundaú & - & 1 & - & 1 \\
\hline São Brás & - & - & 2 & 2 \\
\hline São José da Laje & 1 & - & - & 1 \\
\hline São Luís do Quitunde & - & - & 1 & 1 \\
\hline São Miguel dos Campos & - & 1 & - & 1 \\
\hline Tanque d'Arca & 6 & 1 & - & 7 \\
\hline Taquarana & 2 & - & 4 & 6 \\
\hline Traipu & - & - & 1 & 1 \\
\hline União dos Palmares & - & 1 & - & 1 \\
\hline Total & 12 & 50 & 63 & 125 \\
\hline
\end{tabular}

Fonte: SINAN (2017).

Tabela 2. Notificação de casos em 2017 por município

\begin{tabular}{|c|c|c|c|c|}
\hline Município de notificação & Ignorado/Branco & Urbana & Rural & Total \\
\hline Água Branca & & & 1 & 1 \\
\hline Campo Grande & 7 & 1 & 6 & 14 \\
\hline Capela & & 1 & & 1 \\
\hline Carneiros & & 1 & & 1 \\
\hline
\end{tabular}


Tabela 2. Notificação de casos em 2017 por município (Continua)

\begin{tabular}{|c|c|c|c|c|}
\hline Município de notificação & Ignorado/Branco & Urbana & Rural & Total \\
\hline Coruripe & & 2 & & 2 \\
\hline Dois Riachos & & 1 & & 1 \\
\hline Jaramataia & & 1 & & 1 \\
\hline Joaquim Gomes & & & 1 & 1 \\
\hline Maceió & 1 & 19 & 2 & 22 \\
\hline Maragogi & 2 & 2 & & 4 \\
\hline Marechal Deodoro & & & 1 & 1 \\
\hline Monteirópolis & & & 1 & 1 \\
\hline Olho d'Água Grande & & & 4 & 4 \\
\hline Pão de Açúcar & & 1 & 1 & 2 \\
\hline Santana do Ipanema & & 3 & & 3 \\
\hline São Brás & & & 1 & 1 \\
\hline São Luís do Quitunde & & 1 & & 1 \\
\hline São Miguel dos Milagres & & 1 & & 1 \\
\hline São Sebastião & & 3 & & 3 \\
\hline Traipu & & & 5 & 5 \\
\hline$\overline{\text { Total }}$ & 10 & 37 & 23 & 70 \\
\hline
\end{tabular}

Fonte: SINAN (2017).

Tabela 3. Perfil dos infectados no ano de 2016

\begin{tabular}{lccc}
\hline Faixa Etária & Masculino & Feminino & Total \\
\hline $1-4$ & - & 1 & 1 \\
$5-9$ & 1 & 1 & 2 \\
$10-14$ & 2 & 2 & 4 \\
$15-19$ & 2 & 3 & 5 \\
$20-39$ & 15 & 24 & 39 \\
$40-59$ & 19 & 20 & 39 \\
$60-64$ & 5 & 11 & 16 \\
$65-69$ & 5 & 4 & 9 \\
$70-79$ & 2 & 7 & 9 \\
80 e + & - & 1 & 1 \\
\hline Total & 51 & 74 & 125 \\
\hline
\end{tabular}

Fonte: SINAN Net (2016).

Tabela 4. Perfil dos infectados no ano de 2017

\begin{tabular}{lccc}
\hline Faixa Etária & Masculino & Feminino & Total \\
\hline$<1$ Ano & 1 & 3 & 1 \\
$5-9$ & & 2 & 3 \\
$10-14$ & 3 & 6 & 5 \\
$20-39$ & 8 & 8 & 26 \\
$40-59$ & 18 & 1 & 4 \\
$60-64$ & 3 & 9 & 1 \\
$65-69$ & 1 & 2 & 12 \\
$70-79$ & 3 & 31 & 4 \\
80 e + & 2 & 39 & 70 \\
\hline Total & 39 & & \\
\hline
\end{tabular}

Fonte: SINAN (2017).

Fatores de risco são mais que taxas construídas segundo variáveis coletadas no meio ambiente. Eles se expressam também na rotina diária das populações que vivem em uma região endêmica, cujas práticas e crenças somente podem ser compreendidas quando seus 'mundos' são explorados (Agar, 1994). Não 
podemos esquecer ainda que, quando o comportamento individual é reduzido a aspectos quantificáveis de conduta, está-se enfatizando a necessidade de controle individual, social e moral (Merchán-Hamann, 1995).

O estudo dos fatores de risco foi o referencial metodológico que nos permitiu diagnosticar instantaneamente as variáveis independentes que pudessem estar associadas à transmissão da esquistossomose na localidade. A partir deste perfil epidemiológico, o estudo etnográfico nos direcionou para o conhecimento e o entendimento das relações sociais e culturais que permeiam estas variáveis para que estas passassem a adquirir sentido e coerência diante da realidade endêmica de Alagoas (Jordão et al., 2014). A opção de compreender uma realidade endêmica, utilizando estes métodos de análise, proporcionou-nos uma concepção holística e mais humana do processo, uma vez que o conhecimento dos elementos condicionantes do adoecer observados, quantificados na subjetividade do imaginário popular, foi ampliado, complementado e enriquecido.

O modelo integrado para análise de uma situação endêmica (Jordão et al., 2014), construído com base nestes conhecimentos, mostrou-se capaz de gerar um nível de entendimento dos problemas de saúde de pequenas comunidades a partir do qual é possível apontar na direção das medidas sociais e sanitárias necessárias ao controle da transmissão da esquistossomose.

Como forma de facilitar o entendimento dos resultados abaixo segue os gráficos analíticos detalhando os resultados e porcentagem das notificações.

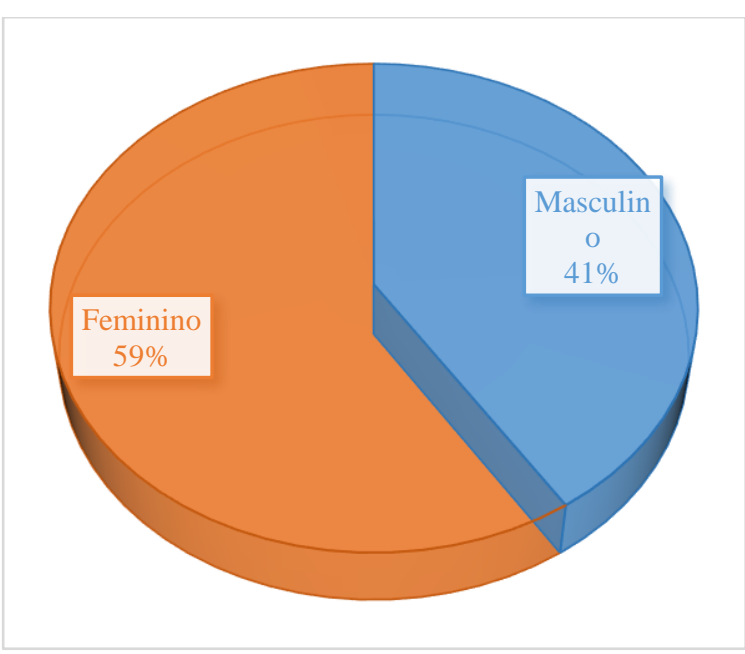

Figura 2. Sexo de casos confirmados 2016

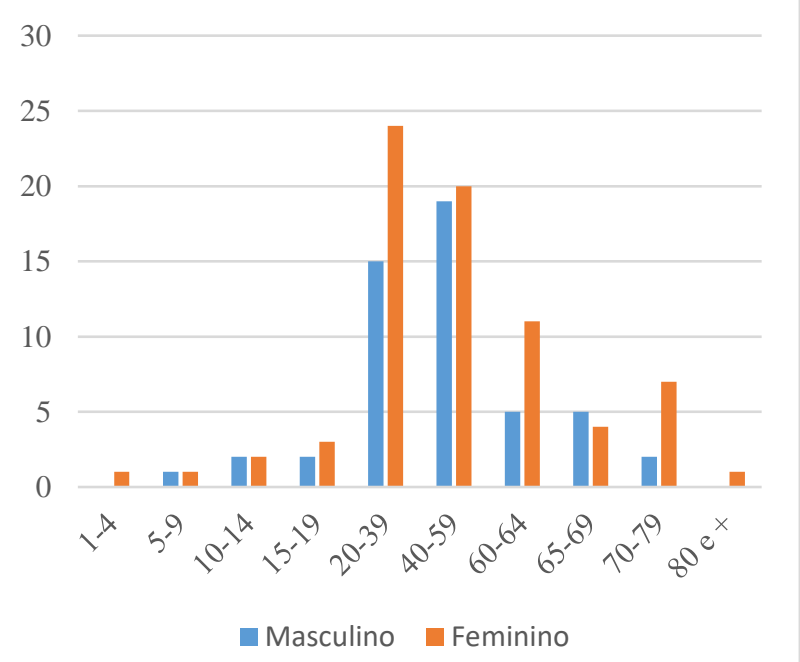

Figura 4. Faixa etária entre homens e mulheres no ano de 2016

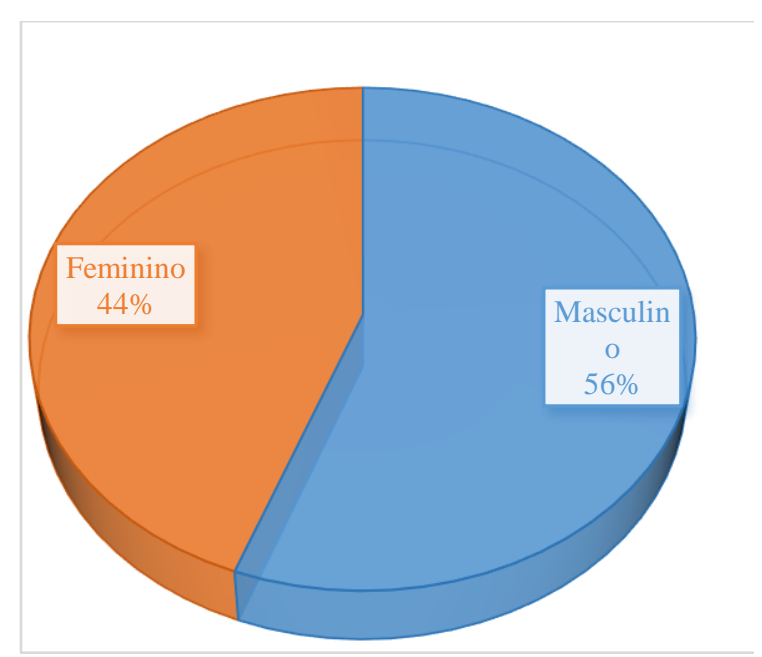

Figura 3. Sexo de casos confirmados 2017

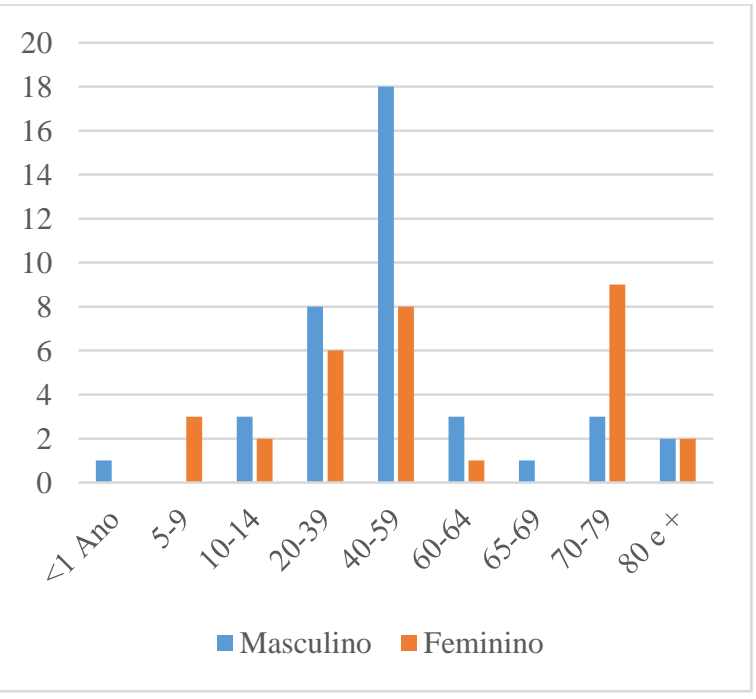

Figura 5. Faixa etária entre homens e mulheres no ano de 2017 


\section{Considerações finais}

Características culturais e econômicas levam a população alagoana a altas condições de risco, pois grande parte não possui água tratada disponível para consumo ou uso geral além de suas residências não possuírem saneamento básico adequado, é de grande importância utilizar medidas preventivas e no controle dos fatores de risco ambientais e educacionais para que possam reduzir a quantidade de casos de esquistossomose mansônica.

Com base no exposto, a esquistossomose não deve ser entendida como um fenômeno biologicamente isolado e sim como uma endemia que tem profundas relações com a cultura, classes sociais e o meio ambiente e com a maneira de viver dos indivíduos tornando-o vulneráveis.

\section{Referências bibliográficas}

Agar, M. (1994). Recasting the "ethno" in "epidemiology". Medical Anthropology, 16(1-4):391-403.

Jordão, M. C. C., Macêdo, V. K. B., Lima, A. F. \& Xavier Junior, A. F. S. (2014). Caracterização do perfil epidemiológico da esquistossomose no estado de Alagoas. Caderno de Graduação-Ciências Biológicas e da Saúde-UNIT-Alagoas, 2(2):175-188.

Katz, N. \& Almeida, K. (2003). Esquistossomose, xistosa, barriga d'água. Ciência e Cultura, 55(1):3843.

Merchán-Hamann, E. (1995). Grau de informação, atitudes e representações sobre o risco e a prevenção de AIDS em adolescentes pobres do Rio de Janeiro, Brasil. Cadernos de Saúde Pública, 11463-478.

Pereira, M. G. (1995). Métodos empregados em epidemiologia. Rio de Janeiro: Guanabara Koogan.

Rocha, T. J. M., Santos, M. C. S., Lima, M. V. M., Calheiros, C. M. L. \& Wanderley, F. S. (2016). Aspectos epidemiológicos e distribuição dos casos de infecção pelo Schistosoma mansoni em municípios do Estado de Alagoas, Brasil. Revista Pan-Amazônica de Saúde, 7(2):6-6.

Saucha, C. V. V., Silva, J. A. M. \& Amorim, L. B. (2015). Condições de saneamento básico em áreas hiperendêmicas para esquistossomose no estado de Pernambuco em 2012. Epidemiologia e Serviços de Saúde, 24497-506.

Secretaria de Saúde do Estado de Alagoas (SESAU) 2012. Dados epidemiológicos referentes a esquistossomose dos anos de 2012. Governo de Alagoas.

SINAN. Disponível em: <http://tabnet.datasus.gov/br/cgi/defthtm.exe?sinnet/cnv/esquitoal.def>. Acesso em: 20 de junho de 2018.

Uchoa, E., Barreto, S. M., Firmo, J. O. A., Guerra, H. L., Pimenta Jr, F. G. \& Costa, M. F. F. L. (2000). The control of schistosomiasis in Brazil: an ethno-epidemiological study of the effectiveness of a community mobilization program for health education. Social Science \& Medicine, 51(10):15291541.

Vitorino, R. R., Souza, F. P. C., Costa, A. P., Faria Júnior, F. C., Santana, L. A. \& Gomes, A. P. (2012). Esquistossomose mansônica: diagnóstico, tratamento, epidemiologia, profilaxia e controle. Revista da Sociedade Brasileira de Clínicas Médicas, 10(1):39-45.

Recebido: 30 de junho, 2019.

Aprovado: 31 de julho, 2019.

Publicado: 7 de setembro, 2019.

Licenciamento: Este artigo é publicado na modalidade Acesso Aberto sob a licença Creative Commons Atribuição 4.0 (CC-BY 4.0), a qual permite uso irrestrito, distribuição, reprodução em qualquer meio, desde que o autor e a fonte sejam devidamente creditados. 